\title{
COVID-19 als Chance für mehr zeitgemäße Bil- dung in der Unterrichts- und Schulentwicklung? Überlegungen zum Selbstverständnis von Lehrkräften im Wandel
}

\section{Jule Böhmer und Johanna Tewes}

\section{Zusammenfassung des Beitrags}

Die unvorhergesehene Umstellung auf Distanzunterricht im Frühjahr 2020 hat Lehrkräfte dazu herausgefordert, neue Konzepte zur digital gestützten Unterrichtsgestaltung zu entwickeln und neue Formen der kollegialen Vernetzung über digitale Formate befördert. Anhand von Daten aus einer kleinen quantitativen Erhebung unter Hamburger Gymnasial- und Stadtteilschullehrkräften soll im Folgenden gezeigt werden, wie die subjektiven Theorien von Lehrkräften Unterrichts- und Schulentwicklung unter veränderten Bedingungen fördern oder hemmen. Daran anschließend soll diskutiert werden, inwiefern die Erfahrungen mit digitalen Vernetzungsstrategien Perspektiven für zukünftige Praxen der kollegialen Kooperation und Kollaboration zwischen Lehrkräften und Schulen eröffnen und dazu beitragen können, das Schulsystem als Ganzes zu transformieren.

\section{Schlüsselbegriffe: Schulentwicklung • Unterrichtsentwicklung $\bullet$ Kultur der Digitalität $\bullet$ zeitgemäße Bildung $\bullet$ Professionalisierung $\bullet$ Kollaboration $\bullet$ Vernetzung}

Durch die Auswirkungen der unerwartet eingetretenen Covid-19-Pandemie im Frühjahr 2020 wurden die bewährten Grundstrukturen des deutschen Schulsystems fundamental erschüttert und alle Beteiligten vor nie dagewesene Herausforderungen gestellt. Bedingt durch die sich fast wöchentlich ändernden behördlichen Vorgaben und Rahmenbedingungen standen nicht nur die Lehrkräfte, sondern auch die Schulleitungen während der Schulschließung unter einem enormen Entwicklungs- und Entscheidungsdruck, was die Organisation des Fern- und Hybridunterrichts und die Gestaltung bzw. Aufrechterhaltung der Schulkultur auf Distanz anging. So zwang die Situation des Fernunterrichts mit ihren radikal veränderten Lehr-, Lernund Kommunikationsbedingungen die Lehrkräfte dazu, ihre kollegialen Kooperationsaktivitäten unter massivem zeitlichem Druck neu auszurichten. Das zunächst meist notgedrungene „Ausweichen“ auf digitale Formen der Unterrichtsgestaltung und der Kommunikation führte dazu, dass Lehrkräfte in berufsbezogenen Kontexten verstärkt mit den Potenzialen und Bedingungen der Digitalität in Berührung kamen.

J. Böhmer und J. Tewes. COVID -19 als Chance für mehr zeitgemäße Bildung in der Unterrichts- und Schulentwicklung? 
Im Zuge dessen suggerierten im Frühjahr 2021 Statements in den Medien wie „Corona, die Jahrhundertchance für deutsche Schulen“ (Spiegel 11.06.2021), „Die Corona-Krise bietet auch viele Chancen“ (Sueddeutsche 24.04.2020) oder „....der Fernlernunterricht hat die pädagogische Landschaft schon jetzt enorm verändert" (FAZ 21.04.2021), dass die Corona-Krise nachhaltige Schulentwicklungsprozesse in Gang setzen werde.

Schulen sind jedoch u.a. aufgrund der Beteiligung vieler verschiedener Akteursgruppen und deren unterschiedlichen Interessen ein sehr träges und sich nur langsam veränderndes System. Zudem wird die Gestaltung von Unterricht mithilfe technischer Geräte oder das Nutzen eines Lernmanagementsystems oft bereits verkürzt als „digitale Bildung“ oder „digitales Lernen“ bezeichnet. Dies zeigt die tiefgehende Fehlvorstellung, dass durch den Einsatz eines Computers, eines interaktiven Whiteboards oder durch Internetrecherche Unterricht automatisch „digital“ werden würde. Ein Transfer des Status Quo ins Digitale lässt sich als Irrweg sehen, da ein additives Verständnis des Einsatzes digitaler Medien suggeriert, dass „es [sich] [...] beim Einsatz digitaler Medien um etwas Zusätzliches und damit prinzipiell Verzichtbares [handle], das auf der Agenda immer dann nach unten rutscht, wenn scheinbar Wichtigeres behandelt werden muss. Von daher könnte es in Zukunft fruchtbarer sein, didaktisches Handeln von vornherein und selbstverständlich mit dem Einsatz digitaler Medien zu verbinden“ (Reinmann 2015, S. 10).

Aus diesem Grund wird der Begriff „Digitalisierung“ nachfolgend nur in Zusammenhang mit der technischen Ausstattung (Hard- und Software) zur Gestaltung zeitgemäßer Lern- und Lehrprozesse verwendet. Diese wiederum erfordern ein Neudenken und Neugestalten von Schule und Unterricht im digitalen Zeitalter im Sinne einer u.a. auf Ergebnisoffenheit, persönliches Sinnerleben, Austausch und Kollaboration angelegten Lernkultur (vgl. Muuß-Merholz 2019, S. 52-53). Dieser Diskurs ist allerdings unter Lehrkräften noch nicht weit verbreitet und vor allem in reformpädagogischen Schulgemeinschaften virulent.

Vor diesem Hintergrund hat uns interessiert, inwiefern die pandemiebedingten Erfahrungen mit der Digitalität und ihren Praxen Einfluss auf die subjektiven Theorien von Lehrkräften hatten und ob sich Personen, deren Unterrichtsgestaltung die Digitalität mit einbezieht, auch offener für eine Vernetzung zwischen Lehrkräften gezeigt haben.

\section{Zum Zusammenhang von Innovation und kollegialer Kooperation}

In den letzten Jahrzehnten sind Praxen der kollegialen Kooperation als Strategien zur Erzeugung von Innovationen in Schul-, Unterrichts- und Personalentwicklungsprozessen verstärkt in den Blick der schulpädagogischen Forschung genommen worden. ${ }^{1}$ Dies geschah u.a. in Reaktion auf gesellschaftliche und bildungspolitische Paradigmenwechsel, die sich u.a. aus den Forderungen der Anpassung schulischer Bildung an die Ergebnisse der PISA-Studien, Inklusion, Integration oder Digitalisierung ergeben haben (vgl. Boller/Fabel-Lamla/Wischer 2018). So schlagen etwa Berkemeyer, Bos, Manitius und Müthing Netzwerke aufgrund ihres

\footnotetext{
${ }^{1} \mathrm{Da}$ dieser Artikel auf veränderte Selbstkonzepte von Lehrkräften fokussiert, können Kooperations- und Kollaborationsprozesse, die darüber hinaus in multiprofessionellen Teams, unter Einbindung der Schüler- und Elternschaft oder außerschulischer Partner oder zwischen Schulen stattfinden, nicht berücksichtigt werden.
} 
Innovationspotenzials „als professionsgemäße Organisationsform für die Unterrichtsentwicklung“ vor (Berkemeyer et al. 2008, S. 20).

Das an kollegiale Kooperationsformen geknüpfte Leistungs- und Innovationsverständnis lässt sich mit Blick auf den Stand mediengestützter Bildungsprozesse an deutschen Schulen zudem exemplarisch an den Ergebnissen der International Computer and Information Literacy Study (ICILS) zeigen. Hier wird betont, dass die Etablierung und Nutzung kollegialer Kooperationsformen wirksame Faktoren darstellen, um die inhaltliche und methodische Unterrichtsentwicklung an Schulen in Bezug auf das kompetenz- und schülerorientierte Lehren und Lernen mit digitalen Medien erfolgreich zu implementieren und die digitalisierungsbezogene Professionalisierung von Lehrkräften zu sichern (vgl. Eickelman/Bos/Gerick 2019, S. 178). Zudem lasse sich auf organisationsbezogener Ebene auch die Anpassung der Schulen an immer neue technologische Entwicklungen sowie pädagogische Anforderungen durch kollegiale Kooperation und Arbeitsteilung besser bewältigen (vgl. Drossel et al. 2016; Hobbs/Coiro 2016).

Die hier genannten positiven Kooperationseffekte offenbaren aber auch eine ökonomische Sicht auf Schule, wonach sich Lernprozesse nicht nur bei Schüler*innen, sondern auch bei Lehrkräften mit dem Ziel der Effizienzsteigerung formalisieren lassen (vgl. Boller/FabelLamla/Wischer 2018, S. 9). Historische wie aktuelle empirische Befunde zum Stand der Kooperation von Lehrkräften zeigen jedoch, dass sich Kooperation in pädagogischen Kontexten nicht nach gouvernmentalisierten Prinzipien steuern lässt. Vor diesem Hintergrund ist es möglich, dass Lehrer*innen zwar durch Fortbildungsinitiativen, schulische Qualitätsrahmen und Schulgesetze dazu verpflichtet werden, miteinander zu kooperieren. Dennoch sind deutliche Diskrepanzen zwischen theoretisch intendiertem Kooperationsverhalten und der tatsächlichen Art, Qualität und Häufigkeit der kollegialen Kooperation an deutschen Schulen zu konstatieren (vgl. Gräsel/Fussangel/Pröbstel 2006; Richter/Pant 2016; Rothland 2018).

Hinzu kommt, dass die neuen Möglichkeiten der sozialen Netzwerkbildung durch die massenhafte Verbreitung des Internets in der Soziologie und der Medienbildungsforschung auch im Kontext von Digitalität analysiert werden (vgl. Castells 2001, S. 431; Kiefer/Holze 2018) und sich Praktiken der digitalen sozialen Vernetzung unter dem Einfluss der CoronaPandemie allgemein verstärkt haben (vgl. von Gehlen 2020; Rosenfeld 2020).

Da sich berufsbezogenes Handeln als Personenhandeln betrachten lässt, lohnt es sich, auf dieser Folie die subjektiven Theorien von Lehrkräften als wichtige Einflussfaktoren für Widerstände, Veränderungsresistenz, aber auch Offenheit für Neues in den Blick zu nehmen.

\section{Wie subjektive Theorien das Handeln von Lehrkräften beeinflussen}

Das Berufsbild einer Lehrkraft lässt sich mit Merkmalen beschreiben, die Kunter und Pohlmann (vgl. 2009) in folgende drei Gruppen einteilen: kognitive, motivationale und emotionale. ${ }^{2}$ Kognitive Merkmale unterteilen sich weiter in Wissen und Überzeugungen, wobei die subjektiven Überzeugungen und Erwartungen eine herausragende Rolle für die

\footnotetext{
${ }^{2}$ Auf die emotionalen und motivationalen Merkmale kann aus Kapazitätsgründen im Folgenden nicht eingegangen werden.
} 
Ausübung der Lehrtätigkeit - und für die Haltung gegenüber Neuerungen - spielen. Lernund schulbezogene Überzeugungen manifestieren sich durch eigene Schulerfahrungen, die formale Ausbildung und eigene persönliche Erfahrungen (vgl. Richardson 1996, zit. nach Kunter/Pohlmann 2009, S. 272). Sie sind dabei recht stabil und veränderungsresistent (vgl. Schank/Abelson 1977, zit. nach Blömeke/Buchholtz 2017, S. 93). Diese subjektiven Erklärsysteme reichen von Einstellungen zur eigenen Person, zum eigenen Fach, zu einzelnen Schüler*innen bis hin zu subjektiven Theorien zum Lehren und Lernen, die handlungsleitend sind (vgl. Kunter/Pohlmann 2009, S. 267). ${ }^{3}$ Für das konkrete Handeln im Unterricht lässt sich das Konzept der „kognitiven Skripte” nach Schank und Abelson heranziehen. Ein Skript als „a predetermined, stereotyped sequence of actions that defines a well-known situation" (Schank/Abelson 1977, S. 41, zit. nach Blömeke/Buchholtz 2017, S. 97) verstanden, gibt Lehrkräften ein Repertoire fertiger Handlungsabläufe an die Hand, das ihnen durch das automatisierte Vorgehen in unbekannten und bekannten Situationen Sicherheit verschafft. Studien zeigen immer wieder, dass in Lernkontexten erworbenes Wissen häufig nicht in anderen Kontexten oder Situationen angewendet werden kann. Dieses „Wissen-HandelnDilemma" bietet eine Erklärung dafür, warum Inhalte aus Fortbildungen häufig nicht Eingang in die schulische Praxis finden. Da das laufende Unterrichtsgeschehen ein hochkomplexes Gefüge darstellt, greifen Lehrkräfte eher auf altbewährte, bekannte Skripte zurück, als Gefahr zu laufen, mit nicht vorhersehbaren Schwierigkeiten konfrontiert zu werden (Schank/Abelson 1977, S. 41, zit. nach Blömeke/Buchholtz 2017, S. 95). Die Fortbildungsinitiativen der letzten Jahre für das Lehren und Lernen im Zeitalter des digitalen Wandels werden sich folglich nicht zwangsläufig auf das Unterrichtsgeschehen niedergeschlagen und zu einer Erhöhung des Einsatzes digitaler Medien im Unterricht geführt haben.

Diese Befunde zu lerntheoretischen Überzeugungen korrespondieren wiederum mit der empirisch belegten Veränderungsresistenz von Lehrkräften gegenüber Neuerungen besonders auf schulsystemischer Ebene (vgl. u.a. Pajares 1992; Terhart 2013): „so ist davon auszugehen, dass sich Konzepte und Überzeugungen nur dann verändern, wenn zum einen die bisher bestehenden alten Konzepte nicht reichen, um beobachtete Phänomene zu erklären, und zum anderen neue Konzepte zur Verfügung stehen, die plausibel und erklärungsmächtig sind“ (Kunter/Pohlmann 2009, S. 272).

Als typische Argumente von Lehrkräften gegenüber Innovationsvorschlägen zählen laut Terhardt „Dafür habe ich keine Zeit“, „Ich nicht, die anderen!“, „Nicht schon wieder!“, „Das funktioniert eh nicht“, „Ich bin schon zu alt, das sollen die Jüngeren machen“ und „Was habe ich davon?"“ (Terhart 2013, S. 494 f.). Diese Argumente spiegeln einerseits die Ausführungen zu den kognitiven Merkmalen und subjektiven Theorien wider. Andererseits haben Untersuchungen gezeigt, dass Lehrkräfte bis heute eine sehr konservative Lehramtsausbildung durchlaufen, die nur sehr selten zulassungsbeschränkt ist. Lehramtsstudierende verfügen häufig über konservative Persönlichkeitsmerkmale und geraten später in ein berufliches Umfeld, das sich durch eine hohe Sicherheit, wenig Aufstiegsmöglichkeiten, kaum

\footnotetext{
${ }^{3}$ Im englischsprachigen Diskurs wird von teacher beliefs gesprochen.
} 
Hierarchien und keine bzw. eine geringe Fortbildungsverpflichtung auszeichnet (vgl. Kunter/Pohlmann 2009, S. 272).

\section{Autonomie und Zusammenarbeit als konträre Selbstkonzepte im Lehrerberuf?}

Subjektive Theorien wie z.B. das individuelle Verständnis von Kooperation haben auch Einfluss auf das kooperative Handeln von Lehrkräften. In Bezug auf die Untersuchung der Kooperationsbereitschaft wurde zunächst das von Lortie in seiner soziologischen Studie erstmals für den Lehrerberuf beschriebene Autonomie-Paritäts-Muster (vgl. Lortie 1972) als ein zentraler und am nachhaltigsten hemmender Faktor für kollegiale Kooperation identifiziert. Demnach liegen der Unterricht und seine Gestaltung in der Verantwortung der ihn durchführenden Lehrkraft, in die sich folglich niemand von außerhalb einzumischen habe (Autonomie). Im Sinne der Parität fordert dieses Muster zudem die Nicht-Sichtbarmachung von Unterschieden in diesem Kontext und sichert somit die Gleichberechtigung und Gleichbehandlung der Lehrkräfte.

Auf Basis dieses bis heute als charakteristisch für den Lehrerberuf geltenden „Verhaltenssyndrom[s] “ (Boller/Fabel-Lamla/Wischer 2018, S. 11) bescheinigen vor allem ältere Studien zur Schuleffektivitäts- und Schulentwicklungsforschung den Lehrkräften in der Bundesrepublik Deutschland, dass kollegiale Kooperation und verstärkte Zusammenarbeit eher eine untergeordnete Rolle in ihrer auf „Einzelkämpfertum“ und die Verteidigung des oben beschriebenen Autonomieanspruchs ausgelegten beruflichen Praxis einnähmen und sie damit Schulentwicklungsprozesse behinderten (vgl. u.a. Esslinger 2002). Diese Form der negativen Verurteilung der pädagogischen Autonomie gegenüber einem positiv behafteten Bild der Lehrkraft als kooperierendem Teamplayer ist aus guten Gründen kritisiert worden, denn die pädagogische Autonomie stellt eine „notwendige und zu schützende Grundvoraussetzung“ im Lehrerberuf dar (Boller/Fabel-Lamla/Wischer 2018, S. 9), die das eigenverantwortliche Arbeiten in einer lose gekoppelten Expertenorganisation wie der Schule überhaupt erst ermöglicht.

Zudem wird bereits in Lorties Erklärungsmodell deutlich, dass die dort für den Lehrerberuf beschriebene Haltung und Norm nicht pauschal als handlungsorientierende Grundüberzeugungen für Lehrkräfte angenommen werden können, da sie abhängig sind von den organisationsstrukturellen Bedingungen, unter denen der Beruf ausgeübt wird (vgl. Köker 2012, S. 77). In neueren Studien konnte dementsprechend nachgewiesen werden, dass sich Autonomiebestreben und Zusammenarbeit im Lehrerberuf nicht zwangsläufig ausschließen und dass sich das traditionelle Bild der Lehrperson als Einzelkämpfer aus dieser Perspektive nicht mehr aufrechterhalten lässt (vgl. Albisser/Keller-Schneider/Wissinger 2013, S. 15).

\section{Was kommt nach Corona?}

Die Frage ist nun, ob die COVID-19-Pandemie und die Folgen für das Bildungssystem eine so tiefgreifende Veränderung der schulischen Rahmenbedingungen darstellt, dass Lehrkräfte ihre subjektiven Theorien z.B. gegenüber digitalen Tools und Kooperation ändern. Zieht man Kollers Theorie der transformatorischen Bildung für die Analyse des Untersuchungszeitraums (vgl. Koller 2018) heran, müsste die Pandemieerfahrung individuelle Bildungsprozesse auch 
bei Lehrkräften anstoßen. Alltagssprachlich wurde die COVID-19-Pandemie häufig als „Corona-Krise“ betitelt. Koller verwendet den Begriff „Krise“ zur Beschreibung von „Situationen oder Konstellationen, in denen die relative Stabilität eines etablierten Welt- und Selbstverhältnisses in Frage gestellt wird“ (Koller 2018, S. 71). Dies führt dazu, dass „Menschen auf Probleme stoßen, für deren Bearbeitung die etablierten Figuren ihres Welt- und Selbstverhältnisses sich als nicht mehr ausreichend erweisen" (ebd., S. 71) und folglich Krisen Bildungsprozesse initiieren. Dabei ist wichtig, dass „Bildungsprozesse angesichts der Herausforderung durch Fremderfahrungen nicht einfach als Selbst-Bildung verstanden werden können, deren Zentrum das sich bildende Subjekt selber wäre. Transformatorische Bildungsprozesse sind [...] vielmehr als responsives Geschehen zu begreifen, das auf einen Anspruch antwortet, der vom Fremden ausgeht" (ebd., S. 86).

Auch neurophysiologische Erkenntnisse helfen, den menschlichen Umgang mit Krisen als angstauslösende Situationen zu verstehen. Während in der direkten Krisensituation das Gehirn in den Überlebensmodus schaltet, kann die empfundene Angst Veränderungsprozesse auslösen, „wenn das, was ihn anschließend erwartet, seiner inneren Natur besser entspricht als das, was er bisher gemacht hat. Wenn er sich dadurch wieder lebendiger und glücklicher fühlt, als das bisher der Fall war. [...] Um wieder mit sich selbst in Kontakt zu kommen, müssten diese Muster erschüttert, destabilisiert, also in einen inkohärenten Zustand gebracht werden. Erst dann besteht die Chance, dass sich diese, das eigene Denken, Fühlen und Handeln bestimmenden Muster umorganisieren“ (Hüther 2020).

Diesen Annahmen nach wären nach der COVID-19-„Krise“ und den Erfahrungen der Lehrkräfte mit unterschiedlichen Unterrichtsformaten (Fern- und Hybridformen) zwei Szenarien denkbar: Entweder waren diese Erfahrungen Auslöser für eine Neuordnung subjektiver Theorien und kognitiver Skripte, die so nachhaltig wirksam sind, dass sie transformatorische Bildungsprozesse in Gang gesetzt haben. Oder es wäre möglich, dass die Schulschließungen als Ausnahmezustand gesehen werden und die gemachten Erfahrungen nicht als Verbesserung des „alten Status Quo“ wahrgenommen werden.

Die Ausführungen in den vorangegangenen Abschnitten haben Mechanismen aufgezeigt, die die Komplexität von Schulentwicklungsprozessen und den großen Einfluss der subjektiven Theorien von Lehrkräften auf die Schul- und Unterrichtsentwicklung verdeutlichen. Dies kann als Erklärungsansatz dienen, warum die Entwicklung zeitgemäßen Lehrens und Lernens auf schulsystemischer Ebene nur in einem sehr langsamen Tempo voranschreitet.

\section{Ergebnisse der Befragung von Hamburger Lehrkräften}

Im Rahmen einer kleinen empirischen, nicht repräsentativen Studie wurden 51 Lehrkräfte weiterführender Schulen in Hamburg im Sommer 2020 mithilfe eines digitalen Fragebogens befragt. Die teilnehmenden Lehrkräfte wurden über ein von den Autorinnen initiiertes Schneeballsystem gefunden. Der Fragebogen enthielt Fragen zum individuell-professionellen Hintergrund, zu den schulischen Rahmenbedingungen (18 Items), zur didaktisch-methodischen Unterrichtsgestaltung mithilfe digitaler Tools (30 Items) und zur kollegialen Kooperation (32 Items). Die Antwortformate variierten von Multiple Choice zu vierstufigen Häufigkeits- bzw. Zustimmungsskalen. Die Items zur methodischen Ausgestaltung und 
didaktischen Zielsetzung basieren auf den von der KMK vorgeschlagenen digitalen Kompetenzen (vgl. KMK 2017) und den Annahmen des SAMR-Modells (vgl. Puentedura 2010), die Items zur kollegialen Kooperation orientieren sich an der TALIS-Studie (vgl. OECD 2020) und der Studie zur Lehrerkooperation von Richter und Pant (vgl. 2016). Die Items wurden an einer kleinen Stichprobe im Mai 2020 pilotiert. Die Nutzung digitaler Tools und das kollegiale Kooperationsverhalten vor und während der ersten Phase der Schulschließungen im Frühjahr 2020 werden in dieser Untersuchung als Indikatoren für die Bedeutung subjektiver Theorien von Lehrkräften für Unterrichts- und Schulentwicklung betrachtet. Die erhaltenen Antworten über Nutzungshäufigkeit und verwendete Tools, intendierte didaktische Zielsetzungen, kollegiale Kooperationsformate und Wünsche für eine Post-Corona-Schule geben Auskunft darüber, wie subjektive Theorien von Lehrkräften als hemmende oder beschleunigende Faktoren für Schul- und Unterrichtsentwicklung auftreten können. Weiterhin ist davon auszugehen, dass sich unter den befragten Lehrkräften verschiedene Gruppenzugehörigkeiten in Bezug auf ihre subjektiven Theorien beobachten lassen. Das von Rogers (vgl. 1983) ${ }^{4}$ entwickelte Innovationszyklus-Modell bietet sich als Erklärhilfe an.

Die Stichprobe ist in Bezug auf die persönlich-professionellen Merkmale sehr heterogen: Es nahmen mehr Frauen als Männer teil (37:14), sie unterrichteten häufiger an einem Gymnasium als an einer Stadtteilschule, knapp die Hälfte verfügte über eine Berufserfahrung von 1 bis 5 Jahren, die Hälfte von ihnen arbeitete an einer Schule mit einer Schülerschaft in bevorzugter Lage (KESS 5+6) $)^{5}$ und die Fächer der Befragten verteilten sich auf alle Fachdomänen.

Die vorliegenden Daten wurden im ersten Schritt mit quantitativen, deskriptiven Analyseverfahren ausgewertet. Bei der Betrachtung der Ergebnisse ist die sehr geringe Stichprobengröße im Blick zu halten. Die Fragen zur schulischen Ausstattung in Bezug auf digitale Geräte ergab, dass die wenigsten Schulen mit genügend Hard- und Software ausgestattet sind, um Unterricht mit digitalen Tools zu gestalten.

Die Analyse der Items zum Einsatz digitaler Tools und zur kollegialen Kooperation können folgendermaßen zusammengefasst werden:

1) Die Häufigkeit der Nutzung digitaler Tools ist nicht wenig überraschend durch den Fernunterricht signifikant gestiegen. Mit Einsetzen des Fernunterrichts erweiterte sich das Nutzungsspektrum digitaler Tools im Fernunterricht deutlich. Im Fernunterricht wurden

\footnotetext{
${ }^{4} \mathrm{Um}$ zu verstehen, wie Implementierungsprozesse von Innovationen verlaufen, wird auch in den Erziehungswissenschaften auf das Diffusionsmodell des Innovationszyklus von Rogers (vgl. 1983) zurückgegriffen. Es beschreibt, wie Innovationen in einem System Verbreitung finden. Rogers unterscheidet, wie fünf verschiedene Nutzertypen jeweils unterschiedlich auf Innovationen reagieren und sich diese aneignen. Er benennt die Gruppen folgendermaßen: Innovators, Early Adopters, Early Majority, Late Majority und Lagards.

${ }^{5}$ Das Akronym KESS steht für „Kompetenzen und Einstellungen von Schülerinnen und Schülern“. Der Index gibt auf einer Skala von eins bis sechs Auskunft über die sozioökonomische Lage der Schülerschaft. Ein KESS-Index von eins beschreibt eine „stark belastete Lage der Schülerschaft“, ein KESS-Index von sechs kennzeichnet eine „bevorzugte soziale Lage der Schülerschaft“" (Bürgerschaft der Freien und Hansestadt Hamburg 2007, S. 1).
} 
digitale Tools zur Interaktion, Kooperation, gemeinsamen Erstellung von Produkten und zum Üben und Festigen von Inhalten signifikant häufiger von Lehrkräften eingesetzt als vor COVID-19. Vor der Pandemie wurden digitale Tools hauptsächlich zur Recherche von Informationen genutzt. Insgesamt lässt sich feststellen, dass die im Fernunterricht gemachten Erfahrungen zu einer größeren Offenheit gegenüber digitalen Tools geführt haben. Dies zeigt sich u.a. in der größeren Zustimmung zu der Gestaltung von Aufgaben mit digitalen Tools, die sich grundlegend von analogen Aufgabenformaten unterscheiden.

2) In Bezug auf das kollegiale Kooperationsverhalten konnte festgestellt werden, dass sich die befragten Hamburger Lehrkräfte grundsätzlich offen für kollegiale Kooperationsformate zeigen und diese auch institutionell zunehmend verankert sind. Gleichzeitig hat der Einfluss von COVID-19 gezeigt, dass die in den Schulen ausgebildeten Kooperationsstrukturen nicht immer als tragfähig oder verlässlich genug angesehen wurden, um die anstehenden Herausforderungen durch kooperatives Handeln zu lösen. So wurden auf der einen Seite an einigen Hamburger Schulen offenbar institutionell bereits bestehende Kooperationsstrukturen zurückgefahren und auf individueller Ebene Tendenzen bestärkt, die ein „Zurückfallen“ in Denk- und Handlungsmuster des Einzelkämpfertums begünstigt haben. Auf der anderen Seite wurden jedoch vor allem durch den Einsatz digitaler Möglichkeiten auch neue Kooperationsformen an den Schulen ausgebildet und erprobt, die vor allem auf der Unterrichtsentwicklungs-, aber auch auf der Organisationsebene einen schnellen Transfer von Ideen, Wissen, Material und Umsetzungsmöglichkeiten zur Gestaltung des Fernunterrichts eröffnet haben. Darüber hinaus hat sich der Einfluss der außerschulischen Netzwerke als eine das in den Schulen vorhandene Wissen ergänzende und die Kolleg*innen zusätzlich emotional stabilisierende Einflussgröße herausgestellt.

3) Um herauszufinden, ob sich das Nutzungsverhalten und die Einstellungen überindividuell ähneln, wurde eine Clusteranalyse durchgeführt. Diese ergab, dass sich die Lehrkräfte in drei verschiedene Gruppen einteilen lassen. Sie lassen sich in Anlehnung an Rogers Innovationszyklus-Modell (vgl. 1983) als Realo-Zweifler, Enthusiasten und Konservierer bezeichnen. Es kann festgehalten werden, dass weder persönliche Merkmale wie Geschlecht, Alter und Berufserfahrung noch schulische Merkmale wie Schultyp oder Zusammensetzung der Schülerschaft Prädiktoren für eine Gruppenzugehörigkeit sind. Dies lässt vermuten, dass Einstellungen zu digitalen Tools und zur kollegialen Kooperation zu den individuellen subjektiven Theorien zu zählen sind. Während die Enthusiasten angeben, auch in Zukunft z.B. digitale Tools einzusetzen, um auch innovative, sich von analogen Vorgehensweisen grundlegend unterscheidende Aufgabentypen einzusetzen, fürchten die Realo-Zweifler, dass sie zu wenig Zeit haben und die technische Ausstattung weiterhin mangelhaft ist. Allerdings haben auch sie viele neue Funktionsweisen digitaler Medien kennen und schätzen gelernt. Auch in Bezug auf die Häufigkeit kollegialer kooperativer Arbeitsweisen vor und während Corona unterscheiden sich diese drei Gruppen. Lehrkräfte, die der Gruppe Enthusiasten angehören, haben schon vor Corona häufiger kollegiale kooperative Arbeitsweisen angewendet als die Lehrkräfte der Gruppe der Realo-Zweifler und der Konservierer. Während der 
pandemiebedingten ersten Phase der Schulschließungen stieg insgesamt die Kooperationshäufigkeit, die Reihenfolge der drei Gruppen blieb dabei bestehen.

Zusammenfassend zeigen die Ergebnisse, dass die pandemiebedingten Erfahrungen per se wahrscheinlich nicht als „Brandbeschleuniger" für große und schnelle Veränderungen in Schule und Unterricht wirken werden. Vielmehr lässt sich sagen, dass die Einstellungen zum Einsatz digitaler Tools und zur kollegialen Kooperation stark von subjektiven Theorien abhängen und es große Unterschiede in der Lehrerschaft in Bezug auf ihre Veränderungsbereitschaft und ihren Veränderungswillen gibt.

\section{Fazit und Ausblick}

Bedeutsam an der Situation der pandemiebedingten Schulschließungen bzw. hybriden Unterrichtsformate in den Jahren 2020 und 2021 war, dass diese über alle Bundesländer und Schulformen hinweg zur selben Zeit stattfanden. Dies führte dazu, dass Lehrkräfte im gesamten Bundesgebiet vor denselben Problemen, Herausforderungen und Chancen standen und sich infolgedessen entsprechend überregional vernetzen konnten, um durch (neue) digitale Tools synchron und asynchron Lösungen für die unsicheren Bedingungen zu erarbeiten und zur Diskussion zu stellen. Der Rückblick auf den Zeitraum von März 2020 bis Sommer 2021 macht deutlich, wie schnell Lehrkräfte informell und auf freiwilliger Basis die sozialen Vernetzungsmöglichkeiten der Medienkultur genutzt haben, um Formen der kollaborativen Wissensproduktion zum Distanz- und Hybridunterricht in berufsbezogenen (Online-)Netzwerken, wie beispielsweise dem \#twitterlehrerzimmer oder über kollaborative Tools und Apps wie Padlets, Miroboards oder Screencasts, wirksam werden zu lassen. Auf diesem Weg gelangen an Phänomenen der Medienkultur und des Prosumings und Sharings orientierte Konzepte der Gemeinschaftlichkeit, wie sie Felix Stalder als eine zentrale Eigenschaft der Kultur der Digitalität beschrieben hat (vgl. Stalder 2017, S. 129 f.), zunehmend in die Kollegien und werden Teil der Schulkultur. Zwar gab es bereits zuvor Initiativen zur Erstellung von OER $^{6}$-Materialien und die damit verbundene Etablierung einer Kultur des Teilens oder auch Barcamp-Formate, in denen Lehrkräfte durch ihre Expertise hierarchiearm von Teilnehmenden zu Teilgebenden werden, aber der Einfluss dieser auf Gemeinschaftlichkeit und Teilhabe angelegten Praxen hat durch die Pandemieerfahrungen deutlich zugenommen.

Diese gerade erst im Werden begriffenen Transformationsprozesse lassen sich anhand der bisher theoretisierten Handlungspraktiken kollegialer Kooperation innerhalb von Einzelschulen, wie sie beispielsweise in den Stufenmodellen der unterrichtsbezogenen Zusammenarbeit nach Gräsel, Fußangel und Pröbstel (vgl. 2006) oder dem schulbezogenen Qualitätsmodell nach Steinert et al. (vgl. 2006) beschrieben werden, nur schwer fassen.

In Bezug auf die zukünftige Theoretisierung von Prozessen der kollegialen Zusammenarbeit erscheint es daher sinnvoll, zwischen kollegialer Kooperation als einem im deutschen Bildungsdiskurs definitorisch spezifizierten Begriff für Formen der professionellen Zusammenarbeit unter Lehrkräften einerseits und Praxen einer noch nicht definierten oder systematisierten

${ }^{6}$ OER = Open Educational Ressource. 
Kollaboration andererseits zu unterscheiden. Bei Terkessidis findet sich dieser Unterschied wie folgt beschrieben: „Bei Kooperation treffen verschiedene Akteure aufeinander, die zusammenarbeiten und die sich nach der gemeinsamen Tätigkeit wieder in intakte Einheiten auflösen. Kollaboration meint dagegen eine Zusammenarbeit, bei der die Akteure einsehen, dass sie selbst im Prozess verändert werden, und diesen Wandel sogar begrüßen” (Terkessidis 2015, S. 14).

Damit wird deutlich, dass die Bereitschaft zur Kollaboration einerseits beim Individuum liegt, sich die damit verbundenen Lern- und Entwicklungsprozesse aber nicht allein auf individualtheoretischer Ebene begründen lassen, weil sie zudem in einem sozialen Interaktionsund Beziehungskontext stattfinden und damit im Sinne der Netzwerktheorie als relational betrachtet und beforscht werden können (vgl. Häußling 2010). Hierzu soll noch ein Zitat angeführt werden, das eine Lehrkraft im freien Kommentarfeld geäußert hat: „Teamarbeit ist für mich nur insofern produktiv, wie die Teammitglieder eine ähnliche Unterrichtsauffassung vertreten. Dann ist es sehr produktiv. Ist das jedoch nicht gegeben und die Teambildung wird von außen gesetzt, ist Teamarbeit anstrengend und nach meinen Erfahrungen unproduktiv. Interessant wären Teams, die sich an gemeinsamen Interessen schulübergreifend bilden. Dafür wären digitale Formen der Zusammenarbeit erleichternd. Ohne einen gemeinsamen Interessenverbund bleiben Teams reine Verwaltungseinheiten, die auch meines Erachtens nur auf dem kleinsten gemeinsamen Nenner kooperieren können und der wäre die Organisation und Verwaltung von Vorgaben. Gemeinsame Produktion setzt Lust an einem gemeinsamen Denken voraus."

Dementsprechend lässt sich in Bezug auf die systemische Gestaltung postpandemischer Fortbildungs- und Schulentwicklungskonzepte schließen, dass auch das Engagement in Kooperations- und Kollaborationsformaten, sofern es von den Lehrkräften als persönlich fruchtbar und sinnstiftend erlebt werden soll, auf intrinsischer Motivation und bedürfnisorientiertem thematischen Interesse basieren und daher freiwillig gewählt und nicht „Top-down“ verordnet werden sollte. Darüber hinaus sollten auch Schulen als lernende Organisationen darin bestärkt werden, sich stärker als professionelle Netzwerke zu begreifen, die die anstehenden Herausforderungen gemeinsam und schulübergreifend meistern können, indem sie Wissen und Expertise teilen. Dazu müssen weitere Strukturen geschaffen werden, in denen Formen der Kollaboration im Sinne gemeinschaftlicher, hierarchiearmer (mediengestützter) Partizipations- und Interaktionsmöglichkeiten es ermöglichen, in unterschiedlichen formellen und informellen Kontexten Input zu erhalten oder zu geben, sich auszutauschen und bedarfsspezifisch zu vernetzen, Ideen zu schmieden, Feedback zu erhalten und Erfahrungen zu reflektieren. Dies kann jedoch nur gelingen, wenn nicht Formen der Kontrolle und der standardisierten Qualitätssicherung im Vordergrund stehen, sondern Zusammenarbeit, Vertrauen, konstruktives Feedback und eine positive Fehlerkultur. Spannend bleibt in diesem Zusammenhang jedoch die Frage des Belastungs- und Autonomieerlebens im Kontext von erhöhten Partizipations- und Kollaborationsmöglichkeiten, denn je mehr Entscheidungskompetenz und Gestaltungsmöglichkeiten im Rahmen von Schulentwicklungsprozessen auf die Einzelschule oder kollaborativ darin agierende Teams übertragen werden, umso mehr Koordination, Kommunikation und Flexibilität ist von den schulischen Akteur*innen gefordert. 
Die Ergebnisse der Befragung Hamburger Lehrkräfte zeigen außerdem, dass viele Lehrkräfte, die vor der Pandemie nicht zu den innovativen Vordenker*innen gehörten, durch die Pandemie mit Ideen zeitgemäßer Bildung in Berührung gekommen sind. Um zu gewährleisten, dass diese Lehrkräfte auch in Zukunft Unterricht zeitgemäß gestalten, mahnt Eickelmann: „[W]ir wissen aus der Schulentwicklungsforschung, dass schulische Innovationsprozesse zwar kriseninduziert sein können, dass sie aber nur dann nachhaltig sind, wenn der Entwicklungsprozess gesteuert wird, auf Akzeptanz bei den schulischen Akteurinnen und Akteuren trifft und die Rahmenbedingungen in geeigneter Weise gestaltet werden" (Jungkamp 2020). Wie im vorliegenden Beitrag erläutert, müssen zeitgemäße Lehrerfortbildungen v.a. stark mit und an den subjektiven Theorien der Lehrkräfte arbeiten, um die Unterrichtsgestaltung nachhaltig zu verändern.

\section{Literatur}

Albisser, Stefan/Keller-Schneider, Manuela/Wissinger, Jochen (2013): Zusammenarbeit in Kollegien von Schulen unter dem Anspruch von Professionalität. In: Keller-Schneider, Manuela/Albisser, Stefan/Wissinger, Jochen (Hrsg.): Professionalität und Kooperation in Schulen. Beiträge zur Diskussion über Schulqualität. Bad Heilbrunn: Klinkhardt, S. 9-29.

Berkemeyer, Nils/Bos, Wilfried/Manitius, Veronika/Müthing, Kathrin (2008): „Schulen im Team“: Einblicke in netzwerkbasierte Unterrichtsentwicklung. In: Berkemeyer, Nils/Bos, Wilfried/ Manitius, Veronika/Müthing, Kathrin (Hrsg.): Unterrichtsentwicklung in Netzwerken. Konzeptionen, Befunde, Perspektiven. Münster: Waxmann, S. 19-70.

Blömeke, Sigrid/Buchholtz, Christiane (2017): Veränderung von Lehrerhandeln beim Einsatz neuer Medien. Design für die theoriegeleitete Entwicklung, Durchführung und Evaluation einer Intervention. In: Zeitschrift für Theorie und Praxis der Medienbildung, 5 (Jahrbuch Medienpädagogik), S. $91-106$.

Boller, Sebastian/Fabel-Lamla, Melanie/Wischer, Beate (2018): Kooperation in der Schule. Ein einführender Problemaufriss. In: Friedrich Jahresheft (XXXVI), S. 6-9.

Bürgerschaft der Freien und Hansestadt Hamburg (2007): Schriftliche Kleine Anfrage und Antwort des Senats. Betr.: Sozialindizes an Schulen - Berechnungsgrundlagen und Folgen (Drucksache 18/6927). URL: hamburg.de/contentblob/4025272/data/pdf-hh-sozialindex-drucksache-186927.pdf (29.06.2021).

Castells, Manuel (2001): Bausteine einer Theorie der Netzwerkgesellschaft. In: Berliner Journal für Soziologie, 11 (4), S. 423-439.

Drossel, Kerstin/Schulz-Zander, Renate/Lorenz, Ramona/Eickelmann, Birgit (2016): Gelingensbedingungen IT-bezogener Lehrerkooperation als Merkmal von Schulqualität. In: Eickelmann, Birgit/Gerick, Julia/Drossel, Kerstin/Bos, Wilfried (Hrsg.): ICILS 2013 - Vertiefende Analysen zu computer- und informationsbezogenen Kompetenzen von Jugendlichen. Münster: Waxmann, S. $143-167$.

Eickelmann, Birgit/Bos, Wilfried/Gerick, Julia (2019): ICILS 2018 \#Deutschland. Computer- und informationsbezogene Kompetenzen von Schülerinnen und Schülern im zweiten internationalen Vergleich und Kompetenzen im Bereich Computational Thinking. Münster: Waxmann.

Esslinger, Ilona. (2002): Berufsverständnis und Schulentwicklung: ein Passungsverhältnis? Eine empirische Untersuchung zu schulentwicklungsrelevanten Berufsauffassungen von Lehrerinnen und Lehrern. Bad Heilbrunn: Klinkhardt. 
Gräsel, Cornelia/Fussangel, Kathrin/Pröbstel, Christian (2006): Lehrkräfte zur Kooperation anregen eine Aufgabe für Sisyphos? In: Zeitschrift für Pädagogik, 52 (2), S. 205-219.

Häußling, Roger (2010): Zur Verankerung der Netzwerkforschung in einem methodologischen Relationalismus. In: Stegbauer, Christian (Hrsg.): Netzwerkanalyse und Netzwerktheorie. Ein neues Paradigma in den Sozialwissenschaften. Wiesbaden: VS Verlag, S. 65-78.

Hobbs, Renee/Coiro, Julie (2016): Everyone learns from everyone: Collaborative and interdisciplinary professional development in digital literacy. In: Journal of Adolescent \& Adult Literacy, 59 (6), pp. 623-629.

Hüther, Gerald (2020): Was wir der Angst verdanken. In: Faktor Magazin. URL: faktor-magazin. de/was-wir-der-angst-verdanken (12.09.2020).

Jungkamp, Burkhard (Juli 2020): Ein Tablet allein macht noch keinen guten Unterricht. Interview mit Prof. Dr. Birgit Eickelmann. URL: info.schulverwaltung.de/schule-trotzt-corona-Interview-Eick elmann (21.08.2021).

Kiefer, Florian/Holze, Jens (2018): Netzwerk als neues Paradigma? Interdisziplinäre Zugänge zu Netzwerktheorien. Wiesbaden: VS Verlag.

Kunter, Mareike/Pohlmann, Britta (2009): Lehrer. In: Wild, Elke/Möller, Jens Möller (Hrsg.): Pädagogische Psychologie. Berlin/Heidelberg/New York: Springer, S. 261-282.

Koller, Hans-Christoph (2018): Bildung anders denken: Einführung in die Theorie transformatorischer Bildungsprozesse. Stuttgart: W. Kohlhammer Verlag.

Köker, Anne (2012): Bedeutungen obligatorischer Zusammenarbeit von Lehrerinnen und Lehrern. Eine neue Perspektive auf professionelle Lerngemeinschaften. Bad Heilbrunn: Klinkhardt.

Kultusministerkonferenz (KMK) (2017): Strategie der Kultusministerkonferenz „Bildung in der digitalen Welt“. Beschluss der Kultusministerkonferenz vom 08.12.2016. Bonn. URL: kmk.org/fileadmin/Dateien/pdf/PresseUndAktuelles/2017/Digitalstrategie_KMK_Weiterbildu ng.pdf (06.06.2020).

Lortie, Dan C. (1972): Team Teaching. Versuch der Beschreibung einer zukünftigen Schule. In: Dechert, Hans-Wilhelm (Hrsg.): Team Teaching in der Schule. München: R. Piper \& Co. Verlag, S. 37-76.

Muuß-Merholz, Jöran (2019): Aufforderung zum Tanz! Damit neue Medien nicht alte Pädagogiken optimieren. In: Routenplaner \#digitaleBildung. Auf dem Weg zu zeitgemäßem Lernen. Eine Orientierungshilfe im digitalen Wandel, Hamburg: Verlag ZLL21 e.V., S. 50-55.

OECD (2020): TALIS 2018 Results (Volume II): Teachers and School Leaders as Valued Professionals. OECD Publishing. URL: oecd-ilibrary.org/sites/19cf08df-en/1/2/5/index.html?itemId=/ content/publication/19cf08df-en\&_csp_=67e65b72be0b468ed3dac915593716de\&itemIGO=oe cd\&item Content Type=book (06.06.2020).

Pajares, M. Frank (1992): Teachers' Beliefs and Educational Research: Cleaning Up a Messy Construct. In: Review of Educational Research, 62 (3), S. 307-332.

Puentedura, Ruben P. (2010): SAMR and TPCK: Intro to Advanced Practise, 2010. URL: hippasus.c om/resources/sweden2010/SAMR_TPCK_IntroToAdvancedPractice.pdf (06.06.2020).

Reinmann, Gabi (2015): Studientext Didaktisches Design. Hamburg: Universität Hamburg. URL: gabi-reinmann.de/wp-content/uploads/2013/05/Studientext_DD_Sept2015.pdf (29.06.2021).

Richter, Dirk/Pant, Hans. A. (2016): Lehrerkooperation in Deutschland. Eine Studie zu kooperativen Arbeitsbeziehungen bei Lehrkräften der Sekundarstufe I. URL: bertelsmann-stiftung.de/filead$\mathrm{min} /$ files/BSt/Publikationen/GrauePublikationen/Studie_IB_Lehrerkooperation_in_Deutschla nd_2016.pdf (06.06.2020). 
Rothland, Martin (2018): „Nie mehr allein“? Was wissen wir über kollegiale Kooperation im Lehrerberuf? In: Friedrich Jahresheft (XXXVI), S. 10-13.

Rogers, Everett M. (1983): Diffusion of Innovations. New York: The Free Press.

Rosenfeld, Dagmar (2020): Das Digitale hält uns jetzt zusammen. In: Die Welt, 17.03.2020. URL: welt.de/debatte/kommentare/article206619163/Corona-Krise-Das-Digitale-haelt-uns-jetztzusammen.html (13.09.2021).

Stalder, Felix (2017): Kultur der Digitalität. Berlin: Suhrkamp.

Steinert, Brigitte/Klieme, Eckhard/Maag Merki, Katharina/Döbrich, Peter/Halbheer, Ueli (2006): Lehrerkooperation in der Schule: Konzeption, Erfassung, Ergebnisse. In: Zeitschrift für Pädagogik, 52 (2), S. 185-204.

Terhart, Ewald (2013): Teacher resistance against school reform: reflecting an inconvenient truth. In: School Leadership \& Management, 33 (5), S. 486-500.

Terkessidis, Mark (2015): Kollaboration. Berlin: Suhrkamp.

von Gehlen, Dirk (2020): Durch Corona wird das Internet zur Selbstverständlichkeit. In: Süddeutsche Zeitung. URL: sueddeutsche.de/digital/coronavirus-internet-chance-digitalisierung-1.4846552 (06.06.2020). 


\section{Informationen zu den Autorinnen}

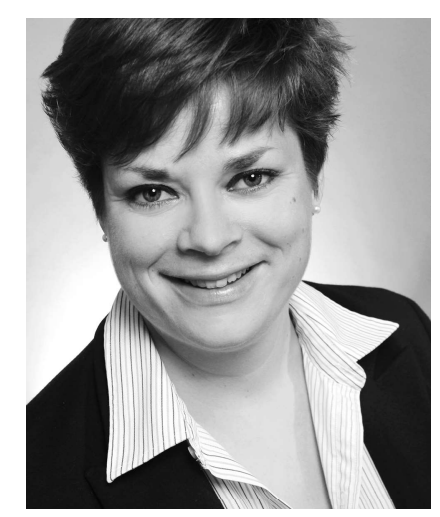

Dr. Jule Böhmer ist zurzeit als wissenschaftliche Mitarbeiterin in der Erziehungswissenschaft an der Universität Hamburg tätig, dort ist sie außerdem Lehrbeauftragte für die Fachdidaktik der Einwanderungssprachen (Russisch/Türkisch). Zudem ist sie Fachreferentin für das Fach Russisch in der Hamburger Behörde für Schule und Berufsbildung. Ihre Forschungsschwerpunkte sind Sprachbildung im Fach, Mehrsprachigkeit und Lehren und Lernen in einer Kultur der Digitalität.

jule.boehmer@uni-hamburg.de

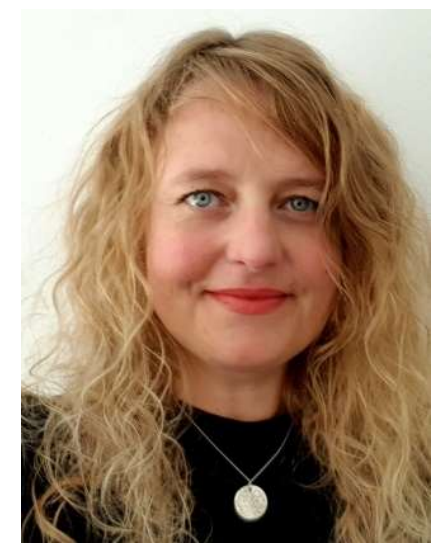

Dr. Johanna Tewes ist derzeit in Hamburg tätig als Lehrerin am Walddörfer-Gymnasium und als Referentin in der Stabsstelle Digitalisierung der Behörde für Schule und Berufsbildung. Zuvor studierte sie Kunst, Deutsch und Geschichte auf Lehramt an der Universität Paderborn sowie den Masterstudiengang „Schulmanagement und Qualitätsentwicklung" an der Christian-Albrechts-Universität zu Kiel. Zudem war sie als wissenschaftliche Mitarbeiterin an der Universität Paderborn und als Lehrbeauftragte an der Universität Hamburg tätig. Ihre Forschungsschwerpunkte sind: Schul- und Unterrichtsentwicklung unter Bedingungen der Digitalität, Historische Kunstpädagogik, Postdigitale Jugendkultur und ästhetische Sozialisation.

johanna.tewes@uni-hamburg.de

\section{Zitationshinweis:}

Böhmer, Jule/Tewes, Johanna (2021): COVID-19 als Chance für mehr zeitgemäße Bildung in der Unterrichts- und Schulentwicklung? Überlegungen zum Selbstverständnis von Lehrkräften im Wandel. In: OnlineMagazin Ludwigsburger Beiträge zur Medienpädagogik, Ausgabe 21/2021. URL: medienpaed-ludwigsburg.de/ 